\title{
Chemical Principles for Electroactive Metal-Organic Frameworks
}

\author{
Aron Walsh*, Keith T. Butler and Christopher H. Hendon
}

Metal-organic frameworks (MOFs) are porous ordered arrays of inorganic clusters connected by organic linkers. The compositional diversity of the metal and ligand, combined with varied connectivity, has yielded over 20,000 unique structures. The application of electronic structure theory can provide deep insights into the fundamental chemistry and physics of these hybrid compounds and identify avenues for design of new multi-functional materials. In this article a number of recent advances in materials modelling of MOFs are reviewed. We present the methodology for predicting the absolute band energies (ionization potentials) of porous solids in comparison to standard semiconductors and electrical contacts. We discuss means of controlling the optical band gaps by chemical modification of the organic and inorganic building blocks. Finally, we outline the principles for achieving electroactive MOFs and outline the key challenges to be addressed in the coming years.

Keywords: metal-organic frameworks, density functional theory, workfunction, bandgap engineering, semiconductor

\section{Introduction}

Atomistic materials modelling has become a valuable tool in contemporary materials science. The accelerated characterization of known materials and the assessment of hypothetical systems is being supported by developments in software and hardware, including an international supercomputing infrastructure and a growing number of reliable simulation packages ${ }^{1}$. The predictive power of numerical simulation approaches, based on a quantum mechanical description of solids, underpins the emerging field of computational materials design ${ }^{2}$.

Whilst first-principles methodologies, e.g. based on density functional theory (DFT), were once limited to simple structures and compositions with 10s 
of atoms in a crystallographic unit cell, modern computer architectures can support the direct simulation of 1000s of atoms. The chemical and physical properties of metal-organic frameworks (MOFs) are now accessible to highquality quantum mechanical simulations. In the past, simple empirical potentials proved useful for screening compositions and topologies in the context of gas storage $^{3}$. As the interest in MOFs extends towards their physical properties and chemical reactivity, knowledge of electronic structure is essential.

In this article, following a brief description of the common workflow for calculating the physical response functions of hybrid solids, we review recent progress in our understanding of the chemical bonding underpinning the electronic structure of MOFs. This includes the atomic and molecular orbitals that overlap to form the valence and conduction bands, the electron addition and removal energies, and set of design principles for tailoring the electronic and optical activity towards functional devices.

\section{From computer to properties}

\section{Input: crystal structure}

A reliable structure is an essential starting point for quantum mechanical calculations of crystalline solids. The ground-state distribution of electrons is determined for a particular arrangement of ions in a lattice that extends infinitely across three spatial dimensions. The crystallography of MOFs is challenging: many reported structures contain solvent molecules, lattice sites with partial occupancy or missing hydrogen atoms. In addition, high symmetry space groups are usually assigned in the absence of hydrogen (due to their low electron density and weak diffraction intensity). The symmetry is commonly lowered when hydrogens are included, for example, when carbon atoms are replaced by the appropriate $\mathrm{CH}, \mathrm{CH}_{2}$ or $\mathrm{CH}_{3}$ groups. One useful resource is the CORE-MOF database: http://gregchung.github.io/CoRE-MOFs that provides 'simulation ready' structures and has been employed in high-throughput screening projects ${ }^{4}$.

A further concern, which has not been fully explored for MOFs, is that Bragg diffraction provides insights into the (spatial and temporal) average crystal structure, but the local environment may be quite different ${ }^{5}$. For organic-inorganic 
solids, there is the possibility for vibrations, librations and rotations of atoms or molecular units ${ }^{6-9}$. Structural disorder has been recognized as a potentially useful trait in framework materials ${ }^{10}$, but from the point of view of atomistic simulation it has been largely neglected up to this point.

\section{Output: physical properties}

The crystal structures taken from experiment are usually subject to local optimization for a chosen description of the interatomic interactions. The three lattice vectors and all lattice sites are minimized with respect to the external pressure and internal forces on the system. For a modern DFT exchangecorrelation functional (e.g. PBEsol ${ }^{11}$ ), experimental and measured structural parameters usually agree to within several percent.

On the UK supercomputer ARCHER, a high-quality electronic structure calculation of a MOF with several hundred atoms in the unit cell will require up to 48 hours on 24 compute nodes (1152 cores). The high computational cost of performing a quantum mechanical calculation is balanced by the wealth of information that can be accessed, including structural, mechanical, magnetic and optical properties. For example, heats of formation can be used to screen hypothetical compositions ${ }^{12}$, the strength of electron-exchange interactions can be used to predict magnetic critical temperatures ${ }^{13}$, and the composition of the frontier orbitals can be used to explain catalytic activity ${ }^{14}$. 

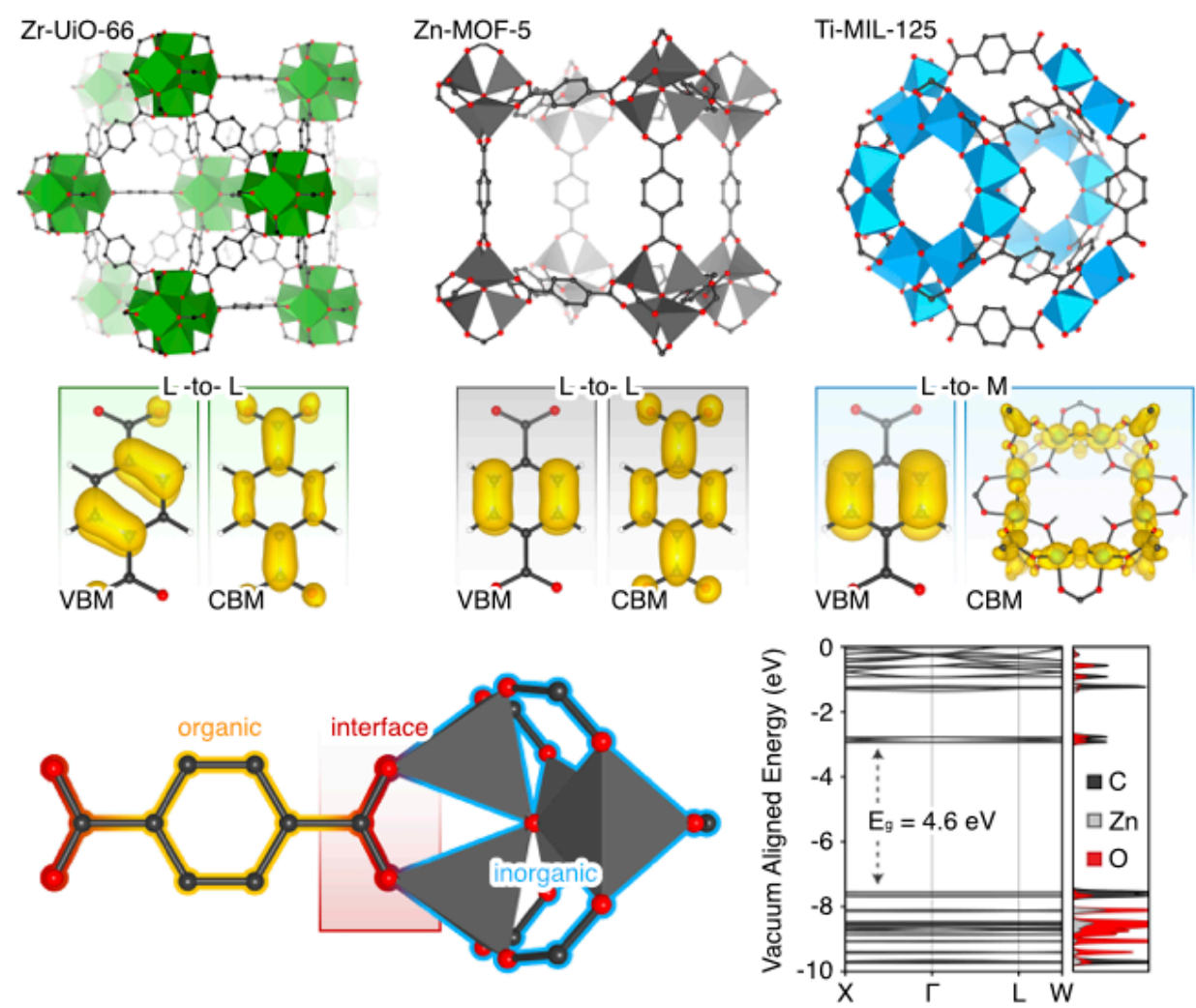

Figure 1. Zr-UiO-66, Zn-MOF-5 and Ti-MIL-125 are composed of closed-shell metal oxide clusters (M) linked by benzene dicarboxylate (L). Their topologies are varied, and are determined by the geometry of the inorganic nodes. The electronic properties and frontier orbital compositions are determined by the interface of the inorganic and organic regions. Most MOFs have poor electronic communication between neighboring motifs, resulting in electronic band structures with low dispersion with charge carrier localization. The electronic band structure (density functional theory with HSE06 exchange and correlation) along the high symmetry lines of of $\mathrm{Zn}-\mathrm{MOF}-5$ is presented in the lower right panel with the atom-projected electronic density of states.

\section{Electronic structure: more than a sum of parts}

A wide variety of metal-organic frameworks have been reported with organic and inorganic networks ranging from $0-3$ dimensions of connectivity ${ }^{15}$. For example, the hybrid halide perovskites that are being intensively studied for applications in solar energy conversion can be considered as the combination of a 3D anionic inorganic framework with a $0 \mathrm{D}$ cationic molecular sublattice ${ }^{16}$. For 
conciseness, we restrict our present discussion to the standard porous MOFs with 3D inorganic-organic-inorganic connectivity, e.g. as found in the ubiquitous MOF-5 that combines a cationic zinc oxide sub-unit connected by anionic benzene dicarboxylate linkers to form a three-dimensional porous framework (see Figure 1).

The orbital composition and spatial location of the valence and conduction bands determines most equilibrium properties of materials. The electronic structure of most MOFs can be described in a manner similar to molecular orbital diagrams common to organometallic chemistry. However, the diverse chemistry of both the organic linkers and the inorganic nodes - for example, whether the node includes an inorganic cation like the $\mathrm{Zn}_{4} \mathrm{O}^{6+}$ cluster of the IRMOF series can require more detailed considerations. In particular, interesting chemistry occurs at the inorganic/organic interface. Whilst studied in detail in the context of surface science and molecular catalysis, the concepts of matching electronic energy levels and orbital symmetry are rarely invoked in MOF chemistry.

An electronic structure calculation can provide insights into the composition, energy and distribution of the frontier extended orbitals (electronic band edges) of any compound. Consider three champion MOFs: $\mathrm{Zr}-\mathrm{UiO}-66,{ }^{17} \mathrm{Zn}$ MOF-5, ${ }^{18}$ and Ti-MIL-125. ${ }^{19}$ Each framework features a closed-shell $\mathrm{s}^{0}$ or $\mathrm{d}^{0}$ metal connected by benzene dicarboxylate linkers. The band edges, however, are the product of the chemistry involving the ligand, metal, and their interface as shown in Figure 1. In the case of Zr-UiO-66 and Zn-MOF-5, both the valence and conduction bands are defined by organic orbitals. Thus, organic functionalization can be used to modify the chemistry of the band edges ${ }^{20,14}$ and the physical properties of the material ${ }^{21}$. Alternatively, the chemically inert $\mathrm{ZrO}_{2}$-based node found in UiO-66 and compositionally similar analogues such as NU-1000 22 can be used to anchor catalytically active metals, providing access to heterogeneous catalysts $^{23}$. Similar metal anchoring is possible through ligand substitutions that boast coordinating functionality (e.g. amines, thiols and alcohols $)^{24}$.

Owing to its wide optical band gap in the UV range $\left(\mathrm{E}_{\mathrm{g}} \sim 4.6 \mathrm{eV}\right)$ and high binding energy $\mathrm{Zn}_{4} \mathrm{O}^{6+}$ derived bands (-8.1 eV below vacuum level - significantly 
deeper than the valence band edge), Zn-MOF-5 offers a further level of chemical modularity allowing for band tuning through both organic funcationalisation, as well as through metal exchange at the Zn-sites. Substitutions for other d-block metals can install mid-gap metal-centered states that result in the MOF featuring a metal-to-ligand transition ${ }^{25}$. Such cationic substitutions have been proposed in the UiO-type materials, although it remains an open question whether the metals exchange or are anchored to the node.

Ti-MIL-125 provides interesting avenues for chemical functionalization because the excitation from the ligand to metal cluster creates a transient Ti(III) center, which is stable for up to $900 \mathrm{ps}^{14}$. Moreover, unmodified Ti-MIL-125 features a band gap $\left(E_{g} \sim 3.8 \mathrm{eV}\right)$ that is tunable with simple organic functionalization ${ }^{26}$.

The chemical modularity of these frameworks reflects their metastability and the chemical softness of the ligand-metal interface. The downside is that many frameworks decomposition in the presence of nucleophiles. Furthermore, weak electronic interaction at their interface results in the flat electronic bands (large effective masses for electrons and holes with $\mathrm{m}^{*}>>1 \mathrm{~m}_{\mathrm{e}}$ ) that are localized in real space. 


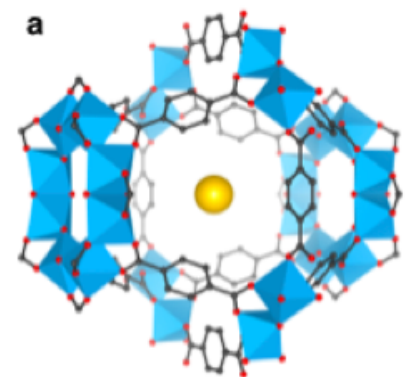

Ti-MIL-125 Sampling Region

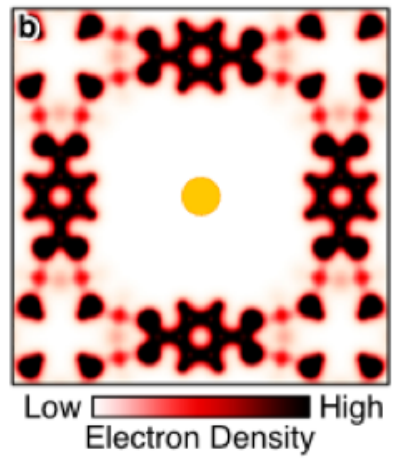

Vacuum Level
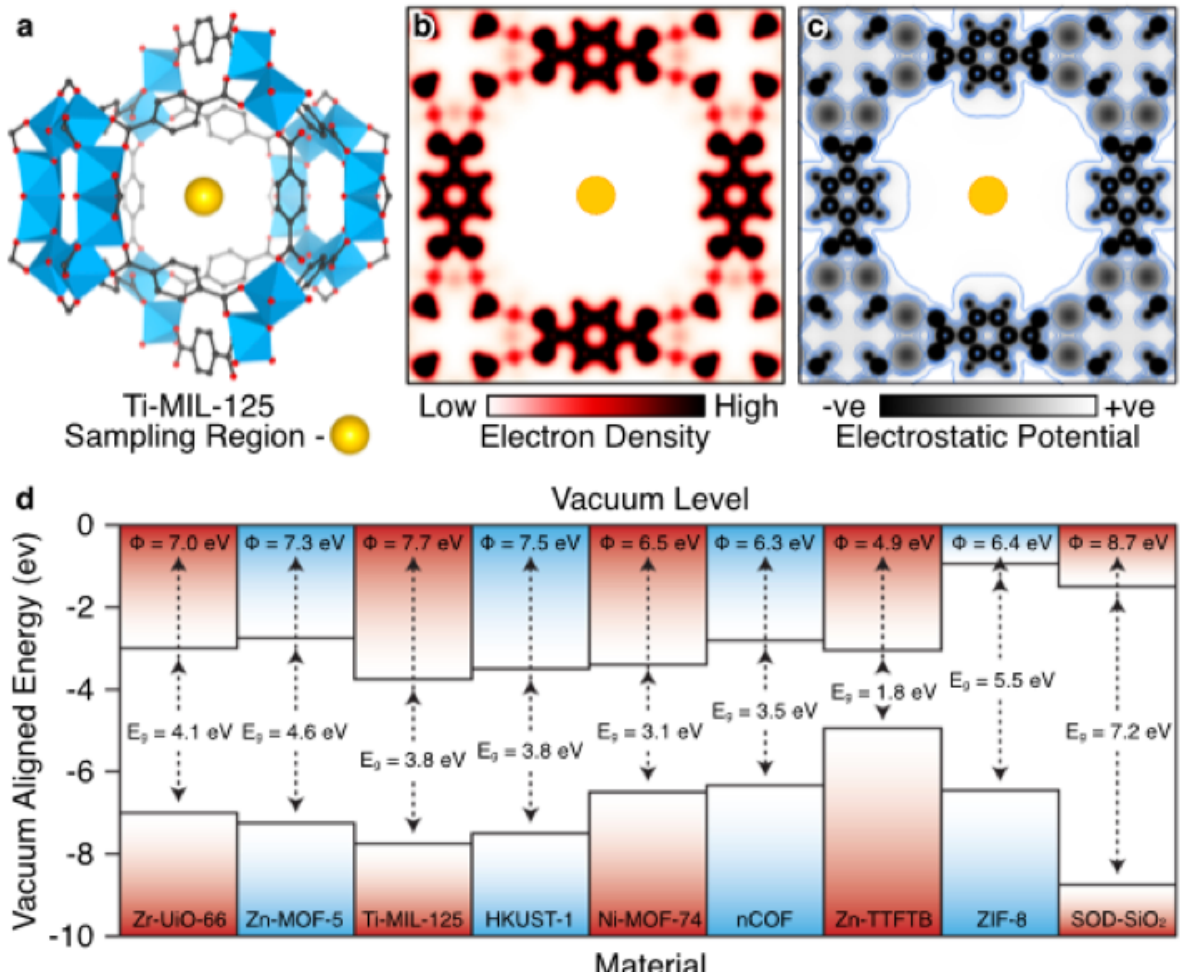

Figure 2. Alignment of the electronic energy levels of metal-organic frameworks adapted from Ref. ${ }^{27}$. (a) Illustration of the internal vacuum level sampling procedure for Ti-MIL-125 along with 2D slices of the electron density (b) and electrostatic potential (c) inside the pore. (d) The calculated valence and conduction band positions for a range of frameworks that are discussed in the main text.

\section{From electron energies to band diagrams}

Knowing the electronic structure of a material can provide useful guidelines for design. The next step, in order to be able to consider a material in a device context, is to place the electron energies on an absolute scale, thus facilitating the construction of energy band diagrams, Figure 2. The construction of band diagrams is a cornerstone of semiconductor device design, as one of the pioneers of heterojunction design Herbert Kroemer famously stated that "If, in discussing a semiconductor problem, you cannot draw an Energy Band Diagram, this shows that you don't know what you are talking about"28.

While ionization potential (electron removal energy) and electron affinity (electron addition energy) are properties that are easily defined for solids, they are 
difficult to quantify. It was recognized as early as the 1930s that the photoelectric threshold of solids is influenced by two factors: the bulk band energies and the surface electrostatic double layer ${ }^{29}$. Due to the sensitivity of the surface term to the history, environment and morphology of a particular sample, there is a large variation in reported values for a given compound.

Similarly for theorists, the use of periodic boundary conditions introduces problems in predicting reliable electron energies. Whilst periodic boundaries offer a convenient and elegant route to representing an infinite crystal from a finite repeating unit, the solution of the Coulomb interactions introduces an arbitrary reference point for the electrostatic potential, meaning that band energies cannot be compared directly between systems ${ }^{30,31,32}$. In another approach the crystal can be modelled by considering a representative cluster of finite size, this provides an external vacuum level and facilitates comparison of band energies between systems $^{33,34}$; however, in practice the models are challenging to construct for multi-component systems.

In this context, we formulated a new procedure to calculate the bulk band energies of porous solids ${ }^{27}$. The method is based on a periodic DFT approach, thereby avoiding truncation problems, and samples the electrostatic potential at the centre of a pore where the electron density has decayed to zero and the electrostatic potential has a plateau (see Figure 2). The procedure, which is applicable to porous solids, is analogous to the use of the external vacuum level above a surface slab as a reference in solid-state calculations. The center of large pores is also a vacuum that provides a reference level for alignment between different materials.

Practically, the alignment is achieved by performing a periodic electronic structure calculation at a given level of theory. The level of theory will affect the reliability of the results and a hybrid exchange-correlational functional (or beyond) is recommended for quantitative insights. The electrostatic (Hartree) density at the centre of the largest pore is then evaluated; this can be achieved using the MacroDensity (https://github.com/WMD-group/MacroDensity) package, which we have developed for this purpose. One must ensure that the 
density has reached a reliable convergence, which is achieved by sampling a large enough area (a sphere of radius $>2 \AA$ ) and ensuing that the variance within the sampling volume is small $(<0.01 \mathrm{~V})$. The Hartree potential thus obtained is now referred to as the vacuum potential $\left(\mathrm{V}_{\mathrm{vac}}\right)$. The ionization potential is simply determined by subtracting the highest occupied band eigenvalue $\left(\mathrm{E}_{\mathrm{VB}}\right)$ : IP $=\mathrm{V}_{\mathrm{vac}}$ $\mathrm{E}_{\mathrm{VB}}$. Similarly, the electron affinity is determined using the lowest unoccupied band eigenvalue $\left(\mathrm{E}_{\mathrm{CB}}\right)$. The resultant ionization potentials and electron affinities can be compared across all materials.

Access to absolute band energies allows for the intelligent design of a range of physical applications for MOFs. Recently the group of Cheng found that using a newly designed ligand they could obtain a material, which electronic structure calculations predict to have band energies suitable for hydrolysis and degradation of organic pollutants $\mathrm{s}^{35}$. Moreover, they experimentally demonstrate the strong photocatalytic activity of the new material. The alignment of energy levels has also been applied to explain the differences in photocatalytic activity in $\mathrm{d}^{0} \mathrm{MOFs}^{14}$. Grau-Crespo and co-workers demonstrated control of frontier orbital positions by metal substitution in porphyrin-based MOFs, allowing crystal engineering for solar fuel production ${ }^{36}$. In the context of device design, access to absolute energy levels as well as lattice parameters from sources such as the CoRE-MOF database will allow for the application of design strategies such as the recently developed ELS (electronic lattice site) metric ${ }^{37}$ allows us to fulfill the conditions of Kroemer's lemma and opens the field for the design of heterojunctions featuring MOFs. 

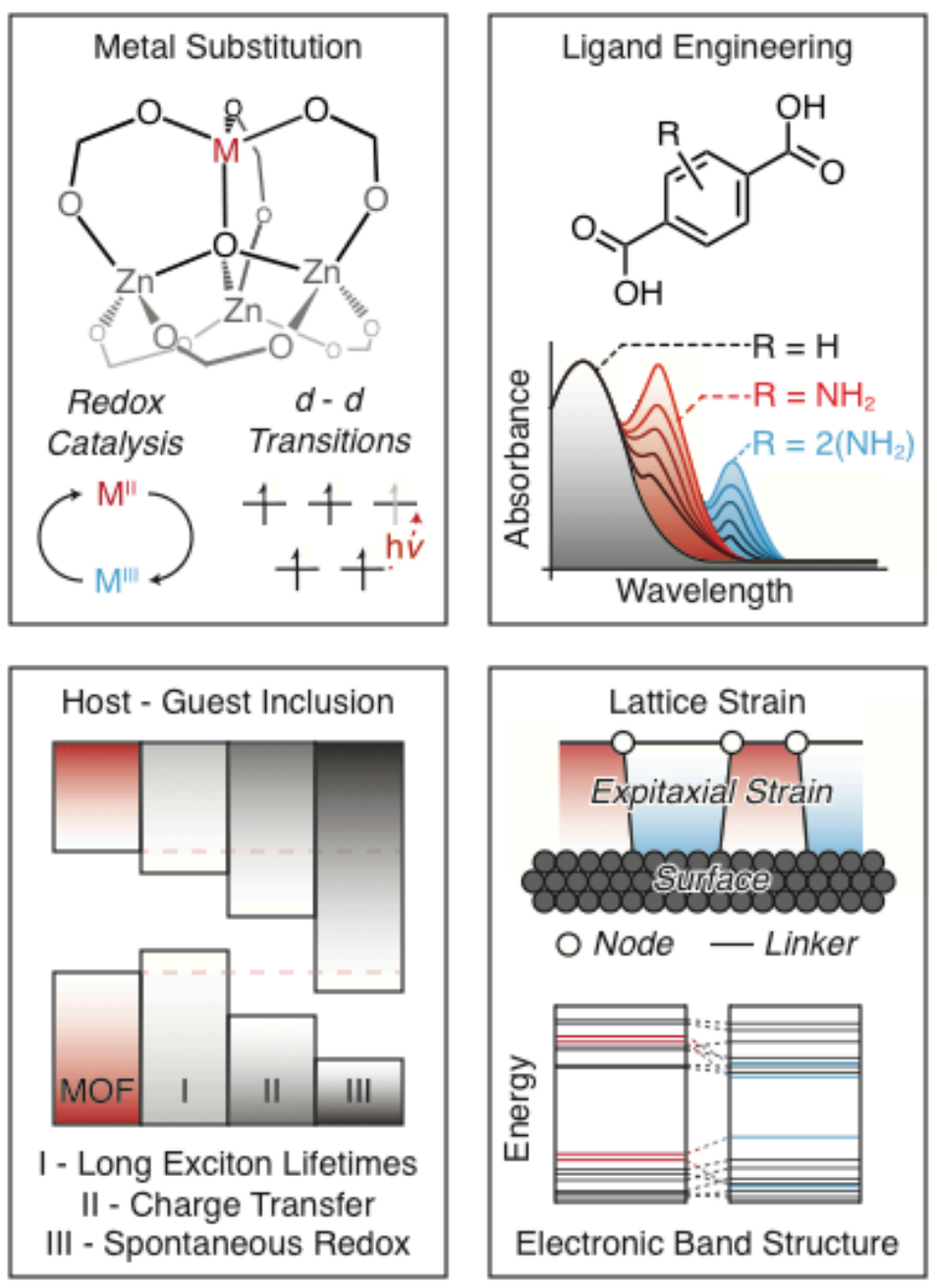

Figure 3. Four avenues for engineering the electronic structure and optoelectronic activity of metal-organic frameworks by modifying the inorganic (metal substitution) and organic (ligand engineering) units, incorporating extrinsic chemical moieties (host-guest inclusion) and mechanical effects from interfaces or embedding (lattice strain). The schematic is inspired by Figure 48 of the seminal nanochemistry perspective by G. A. Ozin ${ }^{38}$.

\section{Principles for electroactivation}

Following our brief outline of chemical bonding in MOFs as well as the factors influencing the band energies, we now collate design principles for tailoring the physical properties. The critical criterion for realizing 
semiconducting MOFs is that the frontier electronic bands are delocalized (low carrier effective masses), which requires effective communication between the organic and inorganic building blocks. However, long-range transport is not always essential, and short-range electron transfer (e.g. to an electrolyte at the surface or penetrating the pore) can be sufficient to support a wide range of redox processes.

A set of four chemical principles for electroactivation of MOFs are outlined in Figure 3 and below. These range from modifying the organic and inorganic building blocks that form the framework to post-synthetic modification and the application of lattice and chemical strain.

\section{Metal substitution}

Depending on the chemical identity and charge state, the orbitals of the metal may form the upper valence or lower conduction band of the MOF. Therefore, metal substitution has the potential to influence both oxidation (hole injection) and reduction (electron injection) processes. For example in HKUST-1 the $\mathrm{Cu} 3 \mathrm{~d}$ orbitals are found at the valence band maximum ${ }^{39}$, while in MIL-125 the Ti $3 \mathrm{~d}$ orbitals are found at the conduction band minimum. Beyond complete metal substitution, the formation of mixed-metal systems offers a promising route to tune redox activity as demonstrated by Brozek and Dinca for MOF- 5 with Ti, $\mathrm{V}, \mathrm{Cr}, \mathrm{Mn}$ and $\mathrm{Fe}$ incorporation ${ }^{25}$.

\section{Ligand engineering}

The choice of ligand can be used to tune the electronic structure directly by modifying the orbital composition or indirectly by changing the framework topology. Many MOFs have at least one frontier band centered on an organic conjugated region. The electron energies of the organic regions are therefore tunable using conventional "push-pull" principles, where the band energies are influenced by the electron donating or withdrawing capability of additional substituents. For example, the introduction of an electron-donating primary amine to a benzene ring will result in an enhancement of electron density and a lowering of the ionization potential. Amination has been effective at introducing visible light photoactivity in a range of simple metal-organic frameworks by raising the 
valence band energy, e.g. modification of benzenedicarboxylayte in MIL-125 ${ }^{26}$ and UiO- $66^{14}$. As an alternative to ligand modification, different types of ligands are being explored, e.g. linear conjugated carboxylates have been shown to exhibit extended helical orbitals ${ }^{40}$ with the potential for long-range magnetic coupling ${ }^{41}$.

\section{Host-guest inclusion}

The introduction of redox active molecular guests into a framework is one way to change the electronic properties, either through spontaneous or lightactivated charge transfer. A related process is the ability to "rewire" an insulating MOF though an auxiliary electroactive linker. The champion system in this regard is HKUST-1 modified with the molecule TCNQ (7,7,8,8tetracyanoquinododimethane) that provided a route to tunable conductivity ${ }^{42}$. In the recent work of Allen and Cohen, cross-linking in a series of isorecticular MOFs was demonstrated ${ }^{21}$, which could be extended to install conductive pathways for a wider range of MOF topologies.

\section{Lattice strain}

A feature of porous frameworks is that they are mechanically soft and flexible in comparison to close-packed materials, which provides another route to engineer their properties. Stress and strain can be introduced in several ways, e.g. epitaxial growth on a rigid substrate, solid-state embedding of MOF particles in a host, the application of pressure either mechanically or chemically (e.g. defects such as missing ligands are sensitive to the growth conditions and reagents $)^{43}$. The electronic structure response is described by the deformation potential: the change in ionization potential, electronic affinity or band gap with respect to a volume (pressure) change. It has been shown that the volume deformation potential are comparable to inorganic semiconductors ${ }^{44}$; however, critically due to smaller bulk moduli the associate pressure coefficients are much larger. In the compound NCOF-1 the band gap pressure coefficient of $-2.2 \mathrm{eV} / \mathrm{GPa}$ is 20 times larger than for bulk $\mathrm{Si}^{45}$. For these materials, a small external stimulus can result in a large change in the electronic structure. 

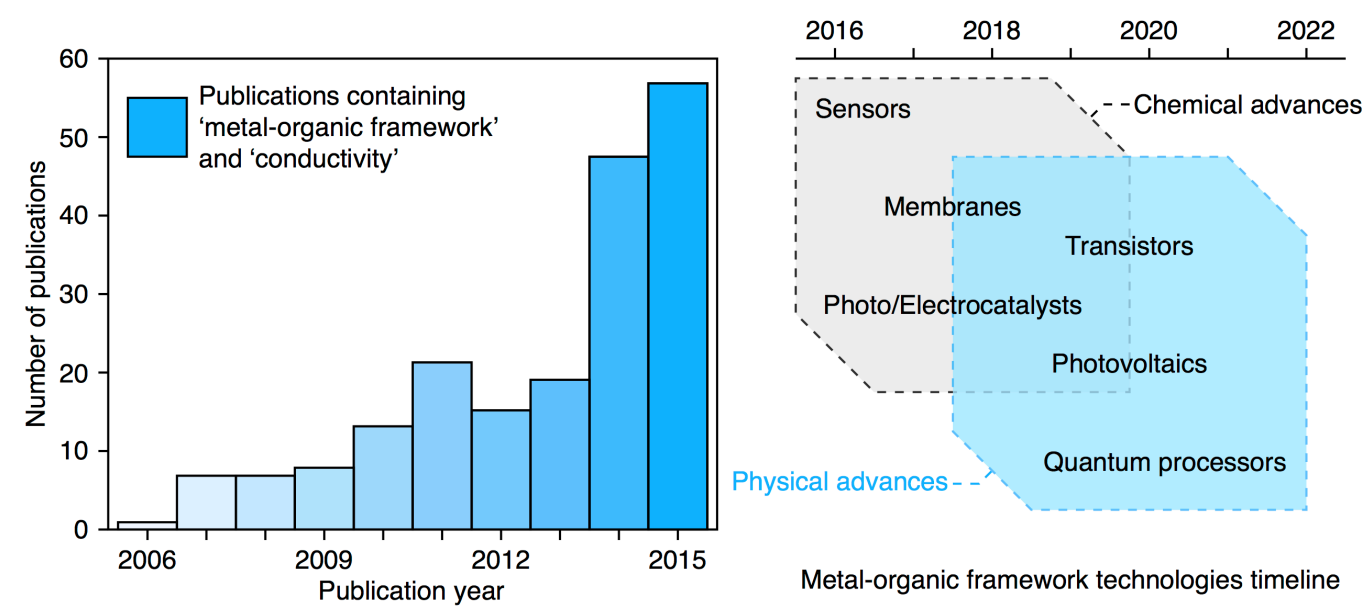

Figure 4. The increase in the number of publications on conductive MOFs (Web of Science, June 2016) with a timeline for future progress for MOF devices building on chemical and physical functionality.

\section{Summary and Outlook}

Our discussion has focused on the successes of modern simulation techniques in describing the chemical bonding and physical properties of metalorganic frameworks. While recent progress has been substantial, the a priori design of functional hybrid solids remains a daunting challenge. We have outlined fertile avenues for the pursuit of materials where the injection, extraction, excitation and transport of charge carriers is controllable, which build on the hybrid compounds reported in this special issue. Not only can the composition of the framework itself be engineered, but the porosity can be exploited for absorbate interactions that activate optical or electronic activity.

The approaches we have outlined for engineering physical properties can be exploited for the use of MOFs in a range of devices, where the hybrid advantage offers the prospect of disruptive technologies. With the number of reports on conductive MOFs rapidly increasing, a roadmap for future progress is illustrated in Figure 4, drawing from the earlier perspective of Allendorf et $\mathrm{al}^{46}$. In Table I we list several possibilities that highlight the potential role for materials modelling. Referring back to the Nobel lecture of Herbert Kroemer: "The 
principal applications of any sufficiently new and innovative technology have always been - and will continue to be - applications created by that technology".

\section{Acknowledgments}

We recognize the contributions of Walter Kohn and Harry Kroto to the development of density functional theory and physical properties of metal-organic framework chemistry, respectively, as well as for stimulating lectures and discussions on these topics. The research discussed here has benefited from collaboration with Jessica K. Bristow, Davide Tiana and Katrine L. Svane. We acknowledge support from the Royal Society, the European Research Council (Grant no. 27757) and the EPSRC (Grant no. EP/M009580/1 and EP/K016288/1).

\section{References}

1. Lejaeghere, K. et al. Reproducibility in density-functional theory calculations of solids. Science (80-. ). 351, 1-11 (2016).

2. Butler, K. T., Frost, J. M., Skelton, J. M., Svane, K. L. \& Walsh, A. Computational materials design of crystalline solids. Chem. Soc. Rev. (2016). doi:10.1039/C5CS00841G

3. Wilmer, C. E. et al. Large-scale screening of hypothetical metal-organic frameworks. Nature Chemistry 4, 83-89 (2011).

4. Chung, Y. G. et al. Computation-Ready, Experimental Metal-Organic Frameworks: A Tool To Enable High-Throughput Screening of Nanoporous Crystals. Chem. Mater. 26, 6185-6192 (2014).

5. Dove, M. T. Theory of displacive phase transitions in minerals. Am. Mineral. 82, 213-244 (1997).

6. Kieslich, G. et al. Role of Amine-Cavity Interactions in Determining the Structure and Mechanical Properties of the Ferroelectric Hybrid Perovskite 
[NH3NH2]Zn(HCOO)3. Chem. Mater. 28, 312-317 (2016).

7. Kieslich, G. et al. Role of entropic effects in controlling the polymorphism in formate ABX3 metal-organic frameworks. Chem. Commun. 51, 1553815541 (2015).

8. Leguy, A. M. A. et al. The dynamics of methylammonium ions in hybrid organic-inorganic perovskite solar cells. Nat. Commun. 6, 7124 (2015).

9. Brivio, F. et al. Lattice dynamics and vibrational spectra of the orthorhombic, tetragonal, and cubic phases of methylammonium lead iodide. Phys. Rev. B 92, 144308 (2015).

10. Cairns, A. B. \& Goodwin, A. L. Structural disorder in molecular framework materials. Chem. Soc. Rev. 42, 4881-93 (2013).

11. Perdew, J. P. et al. Restoring the Density-Gradient Expansion for Exchange in Solids and Surfaces. Phys. Rev. Lett. 100, 136406 (2008).

12. Hendon, C. H., Tiana, D., Vaid, T. P. \& Walsh, A. Thermodynamic and electronic properties of tunable II-VI and IV-VI semiconductor based metal-organic frameworks from computational chemistry. J. Mater. Chem. C 3, 95-100 (2013).

13. Svane, K., Saines, P. J. \& Walsh, A. Magnetoelastic coupling in the cobalt adipate metal-organic framework from quasi-harmonic lattice dynamics. $J$. Mater. Chem. C 3, 11076-11080 (2015).

14. Nasalevich, M. et al. Electronic origins of photocatalytic activity in $\mathrm{d} 0$ metal organic frameworks. Sci. Rep. 6, 23676 (2016).

15. Cheetham, A. K., Rao, C. N. R. \& Feller, R. K. Structural diversity and chemical trends in hybrid inorganic-organic framework materials. Chem. Commun. (Camb). 4780-4795 (2006). doi:10.1039/b610264f 
16. Frost, J. M. et al. Atomistic origins of high-performance in hybrid halide perovskite solar cells. Nano Lett. 14, 2584-2590 (2014).

17. Cavka, J. H. et al. A New Zirconium Inorganic Building Brick Forming Metal Organic Frameworks with Exceptional Stability. J. Am. Chem. Soc. 6, 13850-13851 (2008).

18. Li, H., Eddaoudi, M., O’Keeffe, M. \& Yaghi, O. M. Design and synthesis of an exceptionally stable and highly porous metal-organic framework. Nature 402, 276-279 (1999).

19. Dan-Hardi, M. et al. A new photoactive highly porous titanium (IV) dicarboxylate. J. Am. Chem. Soc. 131, 10857-10859 (2009).

20. Shen, L., Liang, R., Luo, M., Jing, F. \& Wu, L. Electronic effects of ligand substitution on metal-organic framework photocatalysts: the case study of UiO-66. Phys. Chem. Chem. Phys. 17, 117-121 (2015).

21. Allen, C. A. \& Cohen, S. M. Exploration of Chemically Cross-Linked Metal-Organic Frameworks. Inorg. Chem. 53, 7014-7019 (2014).

22. Mondloch, J. E. et al. Vapor-Phase Metalation by Atomic Layer Deposition in a Metal-Organic Framework. J. Am. Chem. Soc. 135, $10294-10297$ (2013).

23. Yang, D. et al. Metal-Organic Framework Nodes as Nearly Ideal Supports for Molecular Catalysts: NU-1000- and UiO-66-Supported Iridium Complexes. J. Am. Chem. Soc. 137, 7391-7396 (2015).

24. Fei, H. \& Cohen, S. M. Metalation of a thiocatechol-functionalized Zr(IV)based metal-organic framework for selective C-H functionalization. J. Am. Chem. Soc. 137, 2191-2194 (2015).

25. Brozek, C. K. \& Dincă, M. Ti(3+)-, V(2+/3+)-, $\operatorname{Cr}(2+/ 3+)-, \operatorname{Mn}(2+)-$, and $\mathrm{Fe}(2+)$-substituted MOF-5 and redox reactivity in $\mathrm{Cr}-$ and Fe-MOF-5. J. 
Am. Chem. Soc. 135, 12886-91 (2013).

26. Hendon, C. H. et al. Engineering the optical response of the titanium-MIL125 metal-organic framework through ligand functionalisation. J. Am. Chem. Soc. 135, 10942-10945 (2013).

27. Butler, K. T., Hendon, C. H. \& Walsh, A. Electronic chemical potentials of porous metal-organic frameworks. J. Am. Chem. Soc. 136, 2703-6 (2014).

28. Herbert Kroemer - Nobel Lecture: Quasi-Electric Fields and Band Offsets: Teaching Electrons New Tricks.

29. Bardeen, J. Theory of the Work Function. II. The Surface Double Layer. Phys. Rev. 49, 635 (1936).

30. Walsh, A. \& Butler, K. T. Prediction of Electron Energies in Metal Oxides. Acc. Chem. Res. 47, 364-72 (2014).

31. Smith, E. Electrostatic Potential at a plane surface of a point ionic crystal. Phys. A Stat. Mech. its Appl. 120A, 327-338 (1983).

32. Ihm, J., Zunger, A. \& Cohen, M. Momentum-space formalism for the total energy of solids. J. Phys. C Solid State Phys. 12, 4409 (1979).

33. Buckeridge, J. et al. Polymorph Engineering of TiO2: Demonstrating How Absolute Reference Potentials Are Determined by Local Coordination. Chem. Mater. 27, 3844-3851 (2015).

34. Scanlon, D. O. et al. Band Alignment of Rutile and Anatase TiO2. Nat. Mater. 12, 798-801 (2013).

35. Wu, Z.-L. et al. A Semi-Conductive Copper-Organic Framework with Two Types of Photocatalytic Activity. Angew. Chem. Int. Ed. Engl. 55, 4938-42 (2016).

36. Hamad, S. et al. Electronic structure of porphyrin-based metal organic 
frameworks and their suitability for solar fuel production photocatalysis. $J$. Mater. Chem. A 3, 23458-23465 (2015).

37. Butler, K. T., Kumagai, Y., Oba, F. \& Walsh, A. Screening procedure for structurally and electronically matched contact layers for high-performance solar cells: hybrid perovskites. J. Mater. Chem. C 4, 1149-1158 (2016).

38. Ozin, G. A. Nanochemistry: Synthesis in diminishing dimensions. $A d v$. Mater. 4, 612-649 (1992).

39. Hendon, C. H. \& Walsh, A. Chemical principles underpinning the performance of the metal-organic framework HKUST-1. Chem. Sci. 6, 3674-3683 (2015).

40. Hendon, C. H., Tiana, D., Murray, A. T., Carbery, D. R. \& Walsh, A. Helical frontier orbitals of conjugated linear molecules. Chem. Sci. 4, 4278-4284 (2013).

41. Tiana, D., Hendon, C. \& Walsh, A. Ligand design for long-range magnetic order in metal-organic frameworks. Chem. Commun. 50, 13990-13993 (2014).

42. Talin, A., Centrone, A. \& Ford, A. Tunable Electrical Conductivity in Metal-Organic Framework Thin-Film Devices. Science (80-. ). 37, 191214 (2013).

43. Bristow, J. K. et al. Free Energy of Ligand Removal in the Metal-Organic Framework UiO-66. J. Phys. Chem. C 120, 9276-9281 (2016).

44. Butler, K. T., Hendon, C. H. \& Walsh, A. Electronic structure modulation of metal-organic frameworks for hybrid devices. ACS Appl. Mater. Interfaces 6, 22044-22050 (2014).

45. Hendon, C. H. et al. Absorbate-induced piezochromism in a porous molecular crystal. Nano Lett. 15, 2149-2154 (2015). 
46. Allendorf, M. D., Schwartzberg, A., Stavila, V. \& Talin, A. A. A roadmap to implementing metal-organic frameworks in electronic devices:

Challenges and critical directions. Chem. - A Eur. J. 17, 11372-11388 (2011). 


\section{Tables}

Table I: Potential applications for electroactive MOFs, with key physical properties and potential for input from materials modelling.

\begin{tabular}{|lll|}
\hline Application & Physical property & Modelling input \\
\hline Photocatalyst & Redox potentials & Band edge positions \\
& Optical absorption & Optical band gaps \\
& Reactivity & Reaction barriers \\
\hline Sensor & Analyte selectivity & Absorption isotherms \\
& Signal processing & Elastic tensors \\
& Deformation potentials \\
\hline Membrane & Sen transport & Diffusion constants \\
& Film quality & Absorption isotherms \\
& Optical absorption & Substrate epitaxy \\
\hline Solar cells & Carrier transport & Effical band gaps \\
& Carrier concentrations & Defect concentrations \\
& Contact resistance & Band edge positions \\
\hline Transistors & Carrier mobility & Effective mass \\
& Film quality & Substrate epitaxy \\
& Contact resistance & Band offsets \\
\hline
\end{tabular}




\section{Author biographies}

\section{Aron Walsh}

Department of Materials, Imperial College London, UK; email a.walsh@imperial.ac.uk

Walsh is Professor of Materials Design at Imperial College London. He received his $\mathrm{BA}$ and $\mathrm{PhD}$ in chemistry from Trinity College Dublin, and subsequently held positions at the National Renewable Energy Laboratory, University College London and the University of Bath. In 2015 he was awarded the EU-40 prize from the European MRS for his work on perovskite solar cells. His research focuses on the theory and simulation of functional materials.

\section{Keith T. Butler}

Department of Chemistry, University of Bath, UK; email k.t.butler@bath.ac.uk

Butler works at the Department of Chemistry at the University of Bath. He received his BA in medicinal chemistry from Trinity College Dublin and $\mathrm{PhD}$ in computational chemistry at University College London. Following postdoctoral research at the University of Sheffield on silicon solar cells, he joined the UK SUPERSOLAR project at Bath to focus on next-generation materials for solar energy conversion.

\section{Christopher H. Hendon}

Department of Chemistry, Massachusetts Institute of Technology, USA; email hendon@mit.edu Hendon is a postdoctoral researcher at the Massachusetts Institute of Technology. $\mathrm{He}$ received his $\mathrm{BSc}$ in chemistry from Monash University and his $\mathrm{PhD}$ in the group of Prof. Aron Walsh at the University of Bath. He maintains a dual interest in the physical chemistry of metal-organic frameworks and the science of specialty coffee. 